\title{
PERTENENCIA DE GÉNERO Y PARTICIPACIÓN POLITICA EN ITALIA
}

\author{
LAURA LEONARDI Y MICHELA BALOCCHI \\ Universidad de Florencia
}

\section{INTRODUCCIÓN}

En el escenario político italiano las mujeres se encuentran prácticamente ausentes. La potencialidad de su presencia, correspondiente a un aumento gradual de la participación de las mujeres en la vida pública, en el acceso a la educación y al mundo profesional, al menos a partir de los años setenta, no se ha visto acompañada por un incremento de su participación en las instituciones, en concreto en los centros de toma de decisión de la política, de la economía, en las organizaciones de partido y sindicales ${ }^{1}$. De hecho, la falta de inclusión de las mujeres en la dimensión política no puede ser separada de la persistencia de una exclusión paralela de las mujeres del ámbito económico y social: en Italia, el desempleo, la pobreza en términos de renta, la precariedad de las condiciones laborales son fenómenos que inciden en especial en el componente femenino de la población. Sin embargo, en el debate público no advertimos atención alguna hacia la cuestión de las desigualdades de género. Un auténtico retraso cultural que se manifiesta también en el atraso del debate científico sobre los indicadores gender sensitive, todavía poco usados en la investigación empírica y en las recopilaciones de datos estadísticos, con repercusiones negativas sobre la posibilidad de interpretar los fenómenos sociales. Y el ámbito de análisis de la participación política -sobre la que concentraremos nuestra atención- no puede eludir esta limitación.

Cabe también decir que, durante mucho tiempo, la escasa participación de las mujeres en los organismos de representación política no ha sido percibida como un "problema" por buena parte de los propios movimientos de las mujeres, quienes desconfiaban de las prácticas institucionales y de las formas de mediación política tradicionales. Algunos grupos punteros dentro del movimiento feminista italiano han teorizado sobre el distanciamiento de las mujeres

1. Gallino, Luciano: Globalizzazione e disuguaglianze, Roma, Laterza, 2000.

Feminismo/s, 3, junio 2004, pp. 31-48 
de la lógica y de los aparatos de la política en sentido estricto, en una búsqueda de formas alternativas de participación, que a menudo encuentran una forma de expresión en el activismo social; pero, de hecho, dichas posturas eluden el problema de la traducción de las nuevas demandas expresadas por las mujeres en nuevas modalidades de gestión y de gobierno de la política.

Por otro lado, las estrategias de lobby (siguiendo el ejemplo anglosajón de la Emily list) adoptadas por las mujeres más cercanas a la política tradicional, dentro de los partidos, dirigidas a poner en marcha un cambio organizativo y de contenidos, no parecen haber tenido un impacto especialmente relevante con respecto a los objetivos prefijados.

El ámbito político de la governance demuestra ser un terreno interesante para indagar las modalidades de encuentro de las distintas culturas de las mujeres -en este caso se habla de "cultura de red»- con respecto a la política, en particular la local. En este contexto las mujeres que ocupan cargos políticos en los espacios de toma de decisión, por ejemplo como consejeras, revelan nuevas maneras de concebir la governance, creando conexiones con las asociaciones formales e informales, con las organizaciones de la sociedad civil, experimentando nuevas prácticas políticas innovadoras por sus formas y contenidos con respecto a la política tradicional. Tal vez sea precisamente a partir de este terreno de análisis que podremos formular algunas hipótesis sobre la posible evolución de la cuestión de la participación política de las mujeres en Italia.

Enfrentándonos, pues, a este tema pasaremos a trazar un cuadro sintético de la presencia femenina y masculina en la política italiana, en los ámbitos nacional y local, con el análisis de los datos relativos a las elecciones políticas de las catorce legislaciones de la República y de algunos datos relativos a las elecciones administrativas locales en los dos últimos turnos electorales; propondremos, además, algunas reflexiones sobre las posibles causas de la diversidad de género existente en los ámbitos nacional y local y sobre algunos de los cambios ocurridos en los últimos treinta años, y concluiremos con algunas consideraciones a raíz de los más recientes fenómenos de renovada participación y movilización política de las mujeres en Italia.

\section{LA DIMENSIÓN NACIONAL DE LA PARTICIPACIÓN POLÍTICA. EL ACCESO DE LAS MUJERES A LAS INSTITUCIONES POLÍTICAS PARLAMENTARIAS}

Desde el nacimiento del Estado Republicano el panorama político italiano ha ofrecido una fuerte disparidad de género en la representación política, así como en la presencia femenina y masculina en los órgano de gobierno, tanto en el ámbito nacional como el local.

La situación es la de una neta infrarrepresentación política de las mujeres que sitúa a Italia en el sexagésimo quinto puesto entre los Parlamentos del mundo por representación femenina, con apenas un 9,6\% de candidatas elegidas en el último turno electoral del 2001 y que ha colocado a Italia en el último 
puesto por presencia femenina en el Parlamento Europeo con un 11,5\% en las elecciones de $1999^{2}$.

La situación es análoga en el ámbito local, donde, de media, en las elecciones administrativas celebradas en 2000 se obtuvo un $8,6 \%$ de candidatas elegidas en los Consejos regionales y un $13,5 \%$ de candidatas nombradas en las Juntas; en este caso, sin embargo, hay que tener en cuenta que nos encontramos ante grandes diferencias regionales, provinciales y municipales.

A este desequilibrio de género en la representación y en la presencia de las mujeres en los órganos de toma de decisión, hay que añadir un atraso cultural y un retraso relativo a los temas inherentes a las cuestiones de género, tanto en el ámbito político-institucional como en el ámbito del debate cultural y científico; y todo esto a pesar de los estímulos provenientes de la actividad del Parlamento Europeo y a pesar de que otros países con problemas similares, como Francia y Gran Bretaña, han abierto un fructífero diálogo filosófico, sociológico, jurídico y político sobre los temas de la representación y de la participación de género.

$\mathrm{Si}$, como apunta Phillips, «la participación igualitaria es uno de los criterios a partir de los cuales son juzgadas las democracias y la infrarrepresentación sistemática de grupos sociales específicos es considerada normalmente como un problema político»" en Italia nos hallamos desde hace más de cincuenta años frente a un problema político, problema que, no obstante, ha sido, en la práctica, mayoritariamente ignorado tanto por los partidos como por los estudiosos de ciencias políticas y sociales y, más en general, por el debate público ${ }^{4}$.

Por otra parte, el problema de una representación de género más equilibrada en la política no se puede reducir-como a menudo ocurre- a un problema de desigualdad dentro de un determinado contexto profesional o laboral, limitando su alcance; y tampoco puede ser concebido como un problema de respeto o negación de la igualdad de oportunidades en sentido formal. Se trata, en cambio, de una auténtica cuestión de inclusión en el área de la ciudadanía social de sujetos que se encuentran en un «estado de minoría» con respecto a las relaciones de poder, un problema que afecta a la representación de los intereses de los ciudadanos, la participación en los asuntos públicos y el buen o mal funcionamiento del sistema democrático de un país.

El análisis de los datos acerca de la presencia de género en el Parlamento italiano desde 1948 hasta ahora, como ya se ha mencionado, muestra una fuerte infrarrepresentación femenina, hasta el punto de que es posible hablar de una auténtica "exclusión» de las mujeres de la representación política hasta

2. Presidenza del Consiglio dei Ministri \& Dipartimento per le Pari Opportunità: Donne in Politica, IV, Roma, Waterloo Comunicazione, 2001.

3. PHILLIPS, Anne: «Democrazia e Rappresentanza. Ovvero, perché il sesso dei nostri rappresentanti dovrebbe avere importanza?», en B. Beccalli (ed.): Donne in quota, Milán, Elementi/Feltrinelli, 1999, p. 156.

4. A propósito del retraso con el que las ciencias sociales y políticas han comenzado a ocuparse de las cuestiones de género en la política, se puede recordar que el primer workshop dedicado a Women and Politics dentro de un congreso científico internacional de ciencias de la política se dio en 1978. 
mediados de los años setenta, o más bien de una «inclusión solamente simbólica», dado que los porcentajes oscilan desde el 3,7\% de candidatas elegidas en la Asamblea Constituyente de 1946 hasta apenas el 2,8\% en 1972 (del cual el $3,7 \%$ en la Cámara y ni siquiera el $1 \%$ en el Senado) 5 . El número de candidatas elegidas empieza a aumentar, aunque de forma discontinua, a partir de la segunda mitad de los años setenta y alcanza su máximo en las elecciones de 1994 con un $13,1 \%$ de mujeres elegidas en la duodécima legislatura, para después bajar de nuevo hasta el 9,6\% de la última jornada electoral de 2001.

Por tanto, se ha dado un aumento de la presencia femenina, pero se trata de un crecimiento muy tenue, que mantiene a las mujeres en un estado de estrecha minoría parlamentaria. Es, además, un crecimiento totalmente inadecuado, si se compara con el que ha sido, en cambio, un aumento significativo de la presencia y de la participación activa de las mujeres en otras áreas de la esfera económica, cultural e institucional del país, ocurrido en los últimos cincuenta años ${ }^{6}$.

Si consideramos los datos sobre las candidatas presentadas y las elegidas en el Parlamento italiano desde 1948 hasta la segunda mitad de los años setenta, además de la ya mencionada desigualdad de género, notamos también un carácter discontinuo tanto en los porcentajes de las candidaturas, como en los de las mujeres elegidas para la Cámara y para el Senado. Los porcentajes oscilan entre el 5,4\% de elegidas de 1948 y el 2,8\% de 1972 (frente el 3,6\% de candidatas en 1948 y el $5 \%$ en 1972$)^{7}$. Se ha interpretado que este movimiento oscilatorio y discontinuo ha sido causado, por un lado, por el desinterés general de los partidos ante el problema de la participación y de la representación política de las mujeres, y por otro lado, por un interés que demuestra ser sólo episódico $y$ tener una finalidad instrumental ${ }^{8}$. También algunos estudios recientes confirman esta tesis respecto a la actitud de los partidos en relación con el voto y la participación política de las mujeres en el período posbélico hasta la mitad de los años setenta, y subrayan, además, cómo se dio una especie de "paternalismo desconfiado», según la definición de Rossi Doria9. Los dos grandes partidos de

5. AnASTASI, Antonino: «Questioni di genere: donna vota donna?», Foedus, 6-III (2003), pp. 60-76.

6. Es suficiente recordar que en una profesión como la de magistrado, a la que las mujeres italianas han tenido acceso sólo en 1965, y a la que se accede por medio de oposiciones y no por cooptación homófila, las mujeres han alcanzado ya el $52 \%$ entre los magistrados de tribunal. Siguen siendo pocas, en cambio, en los ámbitos superiores: entre los magistrados del Tribunal Supremo, por ejemplo, son el $7,4 \%$, pero también en este caso se ha producido un fuerte aumento con respecto a la década anterior, cuando eran apenas el 0,4\% (datos tomados del ISTAT (Instituto Nacional de Estadística Italiano) 2004, www.istat.it).

7. Los datos cuantitativos relativos al Parlamento han sido tomados de ANASTASI, Antonino: Op. cit., p. 36 y de Pupo, Rosaria: «Le donne nel Parlamento italiano dal 1948 al 1996. Un'indagine quantitativa» en D. Barazzetti e C. Leccardi (eds): Genere e mutamento sociale: le donne tra soggettività, politica e istituzioni, Roma, Rubbettino, 2001, pp. 173-180.

8. Por interés instrumental se entiende la atención a exigencias contingentes de «imagen» vinculadas a la competición electoral, gestionadas por las cúpulas de los partidos, y no a un deseo real de representación femenina ni a un interés que responda concretamente a impulsos procedentes desde "abajo» (CotтA, Maurizio: Classe politica e parlamento in Italia, Bolonia, Il Mulino, 1979).

9. Rossi Doria, Anna: Diventare cittadine. Il voto delle donne in Italia, Florencia, Giunti, 1996. 
masa, dominantes en la escena política de la primera república, la Democracia Cristiana (DC) y el Partido Comunista Italiano (PCI), tenían una actitud diferente hacia el voto femenino; ambos, sin embargo, temían que éste pudiera no ser autónomo, y consideraban que las mujeres se dejarían influir en su elección por autoridades extra-políticas, ya fueran éstas las figuras masculinas de la familia (padres, hermanos, maridos) o, en cambio, la Iglesia y los párrocos. La idea que unía a los partidos laicos por un lado y a la DC por el otro era que la elección femenina pudiera verse dictada por un comportamiento conservador, rutinario, vinculado al respeto de la autoridad familiar o religiosa; por consiguiente, los temores hacia los efectos del voto eran opuestos, pero, en ambos casos, se preveía y se daba por descontada una supuesta pasividad del voto femenino, debida al rol tradicional en el seno familiar o al vínculo de pertenencia religiosa ${ }^{10}$.

A pesar de los estereotipos difundidos, las creencias y las preocupaciones de la clase política, el voto femenino resultaba ser, de hecho, autónomo, heterogéneo y problemático, como resulta evidente en análisis más cuidadosos y profundos ${ }^{11}$, y como luego se haría patente a finales de los años sesenta y a principios de los años setenta, con la aparición de nuevas formas de participación política y de nuevos movimientos sociales, entre ellos los específicos de las mujeres, como el movimiento feminista. De esta nueva participación y politización de las mujeres se empiezan a ver algunos efectos en la política institucional a mediados de los años setenta, cuando las mujeres elegidas pasan del 2,8\% de 1972 al 6,7\% de 1976; aún más relevante es, sin embargo, el aumento de las candidaturas, que del $5 \%$ de 1972 pasan a casi el 12\% cinco años más tarde, manteniéndose en ese porcentaje hasta el 14\% y el 16\% de 1987 y 1992 respectivamente.

La cita electoral de 1987 fue significativa desde el punto de vista de la movilización de las asociaciones de mujeres, que se organizaron con el fin esencial de apoyar las candidaturas femeninas y que ejercieron su presión en dos frentes: sobre los partidos, desde el interior de los mismos así como desde el exterior, pidiendo no sólo que se presentara la candidatura de un mayor número de mujeres, sino que se reservara al menos para algunas de ellas unos puestos seguros en las listas; y sobre los medios de comunicación, para obtener visibilidad y para sensibilizar a la opinión pública transmitiendo un mensaje que no pretendía ser sólo un eslogan y una indicación sobre el voto preferente al electorado: «mujer vota a mujer». La campaña de presión obligó a los partidos a presentar la candidatura de más mujeres, pero, de todas formas, fueron los partidos más sensibles a esas exigencias los que se abrieron a las mujeres, es decir, fue sobre todo el PCI, que en la Cámara pasó a un $27,8 \%$ de candidatas elegidas, frente al $4,5 \%$ de la DC y el $5,2 \%$ del Partido Socialista Italiano (PSI) (y frente al $20 \%$ registrado por el mismo PCI en la anterior legislatura). En 1987, por tanto, con

10. Boccia, Maria Luisa: La differenza politica. Donne e cittadinanza, Milán, Il Saggiatore, 2002.

11. Buttafuoco, Annarita: Questioni di cittadinanza. Donne e diritti sociali nell'Italia liberale, Siena, Protagon Editori Toscani, 1997. 
la fuerte movilización de las mujeres por un lado, y la apertura «táctica» por el otro, se supera por primera vez el umbral del $10 \%$ de mujeres elegidas sobre el total (el 10,5\% de elegidas sobre el total; el 12,5\% en la Cámara).

No resulta, en cambio, igualmente positiva la jornada electoral de 1992. Para Italia son años de profunda crisis política. A consecuencia de los casos judiciales (conocidos por las denominaciones de "Manos limpias» y "Tangentopoli») que afectan a eminentes exponentes de los principales partidos italianos en el poder, en particular la DC y el PSI, se derrumba el aparato entero sobre el que se había basado el sistema de gobierno del país durante cincuenta años. La desestructuración de todos los partidos políticos que, a través de alianzas y coaliciones, habían gobernado hasta ese momento, y la deslegitimación de la totalidad de la clase política que había ostentado el poder provocaron el nacimiento de la necesidad de encontrar personas y rostros nuevos que presentar como candidatos. Esta necesidad de cambios propulsó de hecho el reclutamiento de un mayor número de mujeres que hasta entonces habían trabajado en política sin ocupar nunca puestos de relieve, pero este impulso no se tradujo en un resultado electoral igualmente positivo. De hecho, frente a un 18\% de candidatas a la Cámara y un $10 \%$ al Senado, el resultado fue inferior a lo esperado con un total de un $8,8 \%$ de elegidas.

En aquella ocasión, probablemente se produjeron dos efectos contrapuestos sobre las candidaturas electorales de las mujeres. Por una parte, se dio el efecto positivo vinculado a la "cuestión moral», y, por tanto, a la búsqueda de personajes nuevos o, por lo menos, no vinculados al sistema político tradicional: aquí la imagen de las mujeres como posibles agentes del cambio se hacía notar con fuerza y el aumento de las candidaturas femeninas es prueba de ello. Por otra parte, sin embargo, se dio también el efecto opuesto, debido al ingreso en el panorama político de fuerzas políticas nuevas, poco sensibles a las cuestiones de género y poco inclines a promover la participación política de las mujeres (Forza Italia, Alleanza Nazionale y Lega Nord). Como apunta Del Re, el prejuicio de género en la política no desapareció ni siquiera ante las exigencias de cambio y los partidos se orientaron hacia la renovación apostando por la dimensión socio-profesional en la elección de los candidatos -facilitando, por tanto, el ingreso en la política a nuevas categorías profesionales, vinculadas al mundo de la sociedad civil y del empresariado-, pero dejando de lado nuevamente la representación de género como cuestión relevante ${ }^{12}$.

En cambio, se produjo un giro en 1994, por un conjunto causas. En primer lugar, continuaba el proceso de renovación del panorama político y de partidos nacional y se reforzaba la presión por parte de las mujeres, sobre todo dentro de los partidos de izquierdas, a favor de una mayor presencia femenina. En

12. Los tres partidos tienen además sensibilidades diferentes con respecto a la dimensión de género en la política; a propósito de ello, remitimos a DeL RE, Alisa: «I paradossi di genere nella rappresentanza", en F. Bimbi (ed): Differenze e diseguaglianze. Prospettive per gli studi di genere in Italia, Bolonia, Il Mulino, 2003, pp. 215-240. 
segundo lugar, la votación se produjo son un sistema electoral diferente, que combinaba el sistema mayoritario (al $75 \%$ ) con el proporcional (al $25 \%$ ) y que introducía, aunque sólo para la parte proporcional, algunas disposiciones para reequilibrar la representación según las nuevas leyes electorales aprobadas en 1993. Éstas establecían que, en las listas proporcionales para la Cámara, se produjera una alternancia entre los candidatos (mujer/hombre u hombre/mujer) y que, para las elecciones regionales o municipales, ninguno de los dos sexos en lista pudiera ser representado en un porcentaje superior a los dos tercios (tres cuartos para los municipios más pequeños, con menos de 15.000 habitantes).

La discusión y aprobación de las dos leyes no tuvo lugar con un gran debate parlamentario ni tampoco en un clima de discusión y debate público relevante; de todas formas, también a consecuencia de estas normas, la duodécima legislatura vio rozar por primera vez el porcentaje más alto de candidaturas femeninas, es decir, un total de 13,1\% de mujeres elegidas en el Parlamento, el 15,4\% en la Cámara ${ }^{13}$. La Corte Constitucional, dos años más tarde, declaró las leyes de 1993 inconstitucionales por violación de la legislación en materia de paridad de tratamiento, abrogándolas con la sentencia 422, de 22 de julio de 1995. Sin entrar en esta cuestión, nos limitamos a notar el efecto que esta decisión tuvo sobre las jornadas electorales sucesivas: en 1996, las mujeres elegidas en el Parlamento bajaron de nuevo al 10,1\%; cinco años después, en la sucesiva y última jornada del 2001, el porcentaje descendió ulteriormente hasta el 9,6\% ${ }^{14}$. Cabe, además, subrayar que, en estas dos ocasiones, la ausencia de normas que respaldaran una representación política más equilibrada se ha visto unida a un silencio casi total por parte de los grupos de presión en defensa de una mayor participación e implicación de las mujeres.

Los porcentajes de elegidas y nominadas en las dos últimas jornadas electorales, por tanto, disminuyen con respecto a 1994 (y, por razones ya mencionadas, disminuyen sobre todo con el sistema proporcional) y ambos se asemejan en la comparación entre candidatas y elegidas en las dos ramas del Parlamento. Lo que cambia es el color político de la coalición vencedora: en 1996 tenemos una coalición de centro-izquierda con Prodi (a la que siguen dos nuevos Gobiernos encabezados por D'Alema y uno último en 2000 con Amato), en 2001

13. Si se analizan los datos detallados por Anastasi (Op. cit., p. 38), y subdivididos por el tipo de sistema electoral, resulta evidente el papel desempeñado por las citadas leyes de 1993 para reequilibrar la representación: el aumento de las mujeres elegidas en 1994, pues, tuvo lugar con el sistema proporcional, con un $40 \%$ de candidatas y un $33,5 \%$ de elegidas frente el $9,3 \%$ de candidatas y el $9 \%$ de elegidas con el sistema mayoritario, en el que esas leyes no eran válidas. En la jornada electoral sucesiva (1996), cuando esas leyes ya no se encontraban vigentes, la cuota de mujeres elegidas con el sistema mayoritario permanece sustancialmente invariable (en el 9,3\% con un $9,5 \%$ de candidatas), mientras que la cuota de mujeres elegidas con el proporcional, aun siendo superior con respecto al mayoritario, baja al 18,7\% (con un $18 \%$ de candidatas).

14. Cabe recordar que, en el período en el que las dos normas estaban vigentes, se produjeron numerosos intentos de fraude o de uso instrumental de las mismas por parte de los partidos, entre los cuales sobresale el caso clamoroso de dos candidatas de la Lega Nord, quienes, elegidas en junio de 1994, fueron obligadas a dimitir para dejar sitio a dos colegas varones (dimisiones rechazadas dos veces y finalmente aceptadas por la Cámara). 
tenemos la de centro-derecha con Berlusconi. Si la presencia de género en el Parlamento en estas dos ocasiones se mantiene similar, cambia, sin embargo, la composición de la formación gubernamental y de la presencia de género en los Ministerios: las elecciones de varios Primeros Ministros reflejan la distinta sensibilidad de los partidos de referencia y es con los dos Gobiernos D'Alema (1998 y 1999) que se obtiene las más alta presencia de mujeres Ministro alcanzada hasta ahora en Italia, es decir, el $24 \%$, con seis mujeres sobre un total de veinticinco Ministros. El Gobierno Berlusconi, en cambio, reduce la presencia a un $8,7 \%$ con sólo dos mujeres sobre 23 (de las cuales, una en el Ministerio de Educación, Universidad e Investigación Científica, y la otra en el Ministerio para la Igualdad de Oportunidades, ministerio sin presupuesto); y, todavía más, el primer Gobierno Berlusconi (1994) había contado con una única mujer Ministro sobre veintiséis, y siempre en el Ministerio para la Igualdad de Oportunidades ${ }^{15}$.

\section{LA "GEOGRAFÍA» DE LA PARTICIPACIÓN DE LAS MUJERES EN LOS GOBIERNOS LOCALES}

El ámbito regional y local resulta especialmente interesante pues en Italia, quizás más que en los demás países europeos, las oportunidades reales de acceso a instrumentos participativos para las mujeres es distinta según el área geográfica de referencia ${ }^{16}$.

La situación en los organismos legislativos (los Consejos) y en los gobiernos locales (las Juntas) -regionales, provinciales y municipales- es muy diferente y puede variar mucho de región a región. Dada su complejidad, nos limitamos solamente a proporcionar algunos datos relativos a las elecciones regionales de 2000, comparándolos con los de las anteriores elecciones de 1995. En ambos casos, la media nacional es baja: en 1995 tenemos un 13,4\% de mujeres elegidas en los Consejos regionales (es decir, 101 mujeres sobre 754) y un 8,3\% de nominadas en las Juntas (13 sobre 156); cinco años más tarde las medias se invierten,

15. Ya el Gobierno Prodi en 1996 había aumentado la presencia ministerial femenina elevándola a tres mujeres sobre veintidós (el 13,6\%) con respecto a la única mujer del Gobierno anterior. Tras el Gobierno D'Alema, con Amato las mujeres bajan de seis a cuatro representando el $16,6 \%$. Por último, se puede recordar que la primera mujer en cubrir el cargo de Ministro fue Tina Anselmi (como Ministro del Trabajo en el tercer Gobierno Andreotti) en 1976, tras treinta y seis Gobiernos.

16. Se habla de un diferente "contrato de género» en relación a por lo menos cinco macro-áreas geográficas -el Noroeste, el Noreste, el Centro, el Sur y las islas-, caracterizadas por sistemas económico-sociales distintos, en los que se da una división del trabajo social repartida de manera diferente según el género (SANTI, Elena: «Contratti di genere. Un'applicazione e confronti europei», en F. Bimbi (ed): Op. cit, pp. 161-188). También subrayan la diversidad de las macro-áreas los datos ISTAT de 2004 que registran cómo casi todo el aumento de la ocupación femenina de los últimos diez años ha tenido lugar en el Centro-Norte (el $85 \%$ o más, es decir, un aumento de un millón ciento cuatro mil empleadas); en el Sur de Italia, en cambio, ha aumentado el índice de actividad de las mujeres en busca de un empleo (del 33\% al 33,6\%) (vid. www.istat.it). 
las elegidas bajan hasta un 8,6\% (63 sobre 736) y las nominadas suben hasta un $13,5 \%(21 \text { sobre } 155)^{17}$.

Con las nuevas leyes electorales, que prevén que sea el alcalde quien elija directamente a sus concejales, un número importante de regiones ha visto aumentar la presencia de mujeres en las Juntas con las elecciones de 2000: se trata de Umbría (37,5\%), Molise y Toscana (ambas 33,3\%), Campania (27,3\%), las Marcas (25\%), Emilia Romaña (16,7\%), y también Liguria y Lazio (respectivamente $12,5 \%$ y $8,3 \%$ ), a pesar de que en estos dos casos los porcentajes permanezcan por debajo de la media nacional. Si en muchas Juntas regionales las mujeres nominadas aumentan, las elegidas en los Consejos, en cambio, disminuyen o, en algunos casos, se mantienen los porcentajes anteriores ${ }^{18}$. Finalmente, hay dos regiones, Calabria y Apulia, en las que no ha habido ni mujeres elegidas ni nominadas.

Al comparar los Consejos legislativos con las Juntas de gobierno de las distintas regiones, nos encontramos, pues, con notables diferencias en el proceso de la presencia de género, diferencias que se manifiestan también en el interior de las mismas regiones. Lo que es posible observar, de hecho, es que frente a una permanente escasa presencia de las mujeres en los órganos legislativos y de gobierno territoriales, nos encontramos sin embargo con casos de porcentajes femeninos que se vuelven consistentes; este fenómeno se hace especialmente evidente en las Juntas de algunas Regiones, Provincias y Municipios ${ }^{19}$. Los factores que entran en juego son múltiples y de diferente naturaleza.

Algunos/as estudiosos/as han centrado su atención en los efectos que los diferentes sistemas electorales pueden tener en relación al género, llegando a la conclusión de que algunos sistemas -como el proporcional- favorecerían la elección de las mujeres, mientras que otros -como el mayoritario uninominal- las penalizarían. Es conveniente, en cambio, considerar, también a la luz de los desarrollos electorales italianos, locales y nacionales, que la importancia asignada al tipo de sistema electoral tendría que ser relativizada y revisada. Es decir, el mismo tipo de sistema electoral puede dar pie a resultados electorales muy distintos según el contexto en el que tienen lugar las elecciones, según los partidos involucrados en la competición electoral y, sobre todo, según el apoyo que los partidos dan a las candidaturas femeninas, así como según la relevancia que en la competición electoral reviste la propia dimensión de género.

Las candidatas resultan vencedoras al igual que sus colegas varones, al margen del sistema de voto, allá donde reciben un fuerte apoyo, o simplemente igual al de los candidatos varones, por parte de los partidos de referencia, y no

17. Los datos han sido tomados de la publicación de la Commissione regionale pari opportunità donna-uomo y del Consiglio Regionale della Toscana: Donne e politica: una questione di numeri? La Toscana e L'Europa agli inizi degli anni Duemila, 14, Florencia, 2001.

18. Solamente el Véneto registra un aumento del porcentaje de consejeros, debido, sin embargo, a una disminución del número total de consejeros (que pasan de sesenta y cuatro a sesenta) y no a un aumento de las elegidas (Ibíd., p. 26).

19. En Toscana, por ejemplo, la media de mujeres en las Juntas provinciales es del $32,8 \%$ y siete provincias sobre diez tienen el $25 \%$ o más, con el máximo del $50 \%$ en la Provincia de Prato. 
sólo un apoyo formal. Aquí, pues, entran en juego otras variables como la sensibilidad del partido ante la cuestión de la igualdad de género como problema político más general, el rol de los partidos al apoyar o no la igualdad de género dentro de sus propias organizaciones, así como durante la campaña electora $\mathrm{l}^{20}$. Parece, además, relevante el papel desempeñado por las organizaciones femeninas, en el ámbito local y nacional, dentro y fuera de los partidos, y la capacidad de las propias organizaciones de ejercer una presión sobre los órganos de los partidos en los distintos niveles y de obtener visibilidad en los medios de información.

\section{LOS MOTIVOS DE LA FALTA DE PARTICIPACIÓN POLÍTICA DE LAS MUJERES Y LOS SIGNOS DE CAMBIO}

El mundo de la política italiana continúa siendo, pues, fundamental y a veces exclusivamente masculino. Pero ¡cuáles son los motivos de la escasa presencia de las mujeres en la política, sobre todo en las cúpulas de la política, en las estructuras de toma de decisión, en los gobiernos, en los organismos de representación?

Las hipótesis que han sido formuladas son múltiples y hacen referencia esencialmente a tres órdenes de factores que interactúan: la división entre la esfera pública y la privada, con consecuencias de exclusión y autoexclusión de las mujeres; la división del trabajo y la incompatibilidad de los roles; las prácticas discriminatorias. De hecho, los factores de la marginación de las mujeres en la política son múltiples, son factores de tipo cultural y de tipo más estrictamente estructural, que inevitablemente se entrelazan entre sí de distintas formas y maneras.

El factor de tipo cultural sobre el que se ha sido fundamentalmente basada la exclusión primero y la marginación después de las mujeres en la política es el que se encuentra construido sobre la dicotomía público-privado, que, ahondando sus raíces en tiempos lejanos, ha estructurado todo el pensamiento político y filosófico, reafirmándose como dominante en Occidente ${ }^{21}$. La ciudadanía se muestra como neutra, pero el modelo de "ciudadano» encarna la simbología de

20. La representatividad de género no parece tener una correlación con la amplitud de la base de los partidarios de la organización política, como atestigua el pasado de la DC, partido con una amplia base femenina, pero con un número exiguo de mujeres presentes en el Parlamento, como señala Del Re, Alisa: Op. cit., pp. 224-225.

21. La distinción público-privado, de Aristóteles en adelante, ha sido construida sobre la diferenciación dicotómica de lo femenino y de lo masculino, con una operación simbólico-ideológica que ha llegado a hacer coincidir lo público y la esfera pública con lo masculino y la esfera privada con lo femenino, dando una connotación de valor a las dos esferas, o sea ratificando una supremacía simbólico de lo masculino sobre lo femenino y de lo público sobre lo privado. De esta forma, durante siglos las mujeres han permanecidos excluidas de las decisiones políticas y de las cuestiones públicas en todas sus formas; si los hombres eran por definición "públicos» y estaban orientados hacia los negocios y la política, las mujeres estaban destinadas a la vida familiar y doméstica, eran por antonomasia "privadas» y, de este modo, también se veían privadas de las condiciones para ser plenamente ciudadanas (OKIn Moller, Susan: Le donne e la giustizia. La famiglia come problema politico, Bari, Dedalo, 1999). 
lo masculino y se fundamenta sobre la dicotomía sexuada de las dos esferas. El acceso a la ciudadanía para las mujeres, pues, ha tenido lugar a expensas de la eliminación de la diferencia y esto ha conllevado, y aún conlleva, las desigualdades de hecho en la obtención de la plenitud de los derechos civiles, sociales y políticos.

Los efectos de la sexualización dicotómica de las dos esferas han ejercido su influjo incluso después de que el acceso de las mujeres a la vida política ya no se ha encontrado bloqueado ni vedado, de modo que, durante largo tiempo, gran parte de las mujeres no han considerado la participación en la vida política como un instrumento eficaz para promover sus propios intereses de grupo. Desde este punto de vista, el hecho de que la esfera política en Italia, aún ahora, siga estando marcada y estructurada sobre la base de una simbología especialmente masculina y compuesta sobre todo por hombres, la convierte en una dimensión alejada de las vivencias de las mujeres, y puede ser, por tanto, percibida como una "fortaleza» masculina. Por consiguiente, se debe tener en cuenta el "efecto desánimo" sobre la participación política activa de las mujeres, con sus posibles consecuencias como la autoexclusión ya sea de la política tout court, ya sea, una vez dentro del ruedo político, de los puestos de toma de decisión y de las posiciones de dirección.

El reconocimiento público de la diferencia de género $y$, al mismo tiempo, de la igualdad e igual dignidad llega a ser hoy una condición esencial para la obtención de la plena ciudadanía por parte de las mujeres ${ }^{22}$, promoviendo una refundación de la política en su organización y en sus contenidos.

Por otro lado, la propia construcción cultural y simbólica de la dicotomía sexuada público-privado se ha basado en la división de los roles y se ha producido y reproducido en la división del trabajo entre hombres y mujeres, que continúa estructurándose de forma inicua. Y uno de los efectos que no pueden ser omitidos de la actual división desigual tanto del trabajo extra-doméstico, como del trabajo de cuidado dentro de la familia y para la familia, consiste en la menor cantidad de tiempo de la que disponen las mujeres con respecto a los hombres. La fuerte reducción del tiempo disponible para las mujeres para "hacer otras cosas", para dedicarse a otras actividades, las coloca de partida en una posición de desventaja por lo que se refiere a la posibilidad de llevar a cabo una participación plena en la vida social y política ${ }^{23}$.

Sin embargo, cabe señalar que las dificultades reales, inherentes a la conciliación de los distintos roles, a veces pueden ser empleadas y evidenciadas de forma estereotipada para marginar y oponerse a las mujeres que se asoman a

22. Bonacchi, Gabriella y Groppi, Angela (eds): Il dilemma della cittadinanza, Bari, Laterza, 1993.

23. Los últimos datos del informe de las Naciones Unidas demuestran y confirman que en casi todos los países del mundo las mujeres (sumando el trabajo remunerado al no remunerado) siguen trabajando más horas al día con respecto a sus conciudadanos; e Italia, entre los países democráticos, es uno de los que cuentan con el mayor nivel de tiempo de trabajo femenino por encima del masculino: hasta el 128\% frente al 98\% de Dinamarca, al 100\% de Gran Bretaña y a la media del 105\% (UNDP: Human Development Report, Nueva York, Oxford University Press, 2003, p. 326). 
la política. En el panorama europeo las familias italianas ${ }^{24}$ parecen ser aquellas en las que es menor el reparto de las tareas domésticas y de los cuidados, en los que las mujeres están pues, más implicadas en la "doble presencia»: este hecho acaba obstaculizando a las mujeres doblemente, puesto que las perjudica al emprender otras actividades, pero también porque alimenta un imaginario colectivo de "no disponibilidad» para la política, que acaba por verse extendido también a aquellas mujeres que poseen, en cambio, los recursos culturales, económicos y sociales para dedicarse a ello. Como apunta Del Re, los primeros en verse influidos por estos estereotipos son precisamente los gatekeepers de los partidos, o sea los personajes clave en la regulación de los accesos a las estructuras políticas ${ }^{25}$.

Factores culturales y factores sociales, pues, se entrelazan y uno de los más relevantes está constituido por el hecho de que la aún exigua minoría de mujeres italianas que se dedica activamente a la vida política aparece sometida a fenómenos de discriminación directa o indirecta, consciente o inconsciente, dentro del sistema político y de partidos. Es suficiente pensar en el ya mencionado fenómeno de fraude, por parte de las direcciones de partido, de las normas electorales de 1993 para reequilibrar la representación; pero también en el escaso apoyo de las propias candidatas por parte de los partidos durante la campaña electoral, que se explica en la asignación más frecuente de mujeres en los distritos difíciles e inseguros. Y aún más, se toma nota del hecho de que también en los partidos en los que ha habido un aumento de la participación política de las mujeres y un ensanchamiento de la base femenina no se ha producido un aumento proporcional ni de las candidatas ni de las elegidas. Evidentemente, aquí entran en escena los mecanismos de selección de las candidaturas y de los recorridos internos de las carreras, que, en la mayoría de los casos, penalizan a las mujeres.

Asimismo, cabe subrayar, como decíamos anteriormente, que los diferentes partidos expresan también diferentes grados de sensibilidad (o, a veces, de desinterés) con respecto a la cuestión de género en política, ya sea como problema interno en el partido, ya sea como problema político más general. En Italia, como en muchos otros países, tradicionalmente los partidos de izquierdas son los que han demostrado una mayor sensibilidad hacia la cuestión de género ${ }^{26}$. Los datos sobre los porcentajes de mujeres elegidas en el Parlamento en los distintos partidos entre 1948 y 1992 muestran un total del 10,9\% de mujeres

24. SANTI, Elena: Op. cit., p. 177. Otro dato interesante es que los hombres, en una medida muy superior a la de las mujeres, utilizan su tiempo principalmente en market activities (es decir, en actividades de producción dirigidas al mercado) y los italianos más que los demás: así, sólo el $23 \%$ del tiempo de los varones italianos es empleado en "actividades no dirigidas al mercado", frente al $40 \%$ del tiempo de los franceses y de los húngaros y al $32 \%$ del tiempo de los ingleses. Una situación aún más marcada en Japón y en la India (donde, respectivamente, sólo el $7 \%$ y el $8 \%$ del tiempo masculino se dedican a non-market activities) (UNDP: Op. cit., p. 326).

25. Del Re, Alisa: Op. cit., p. 219.

26. Recuérdese, por ejemplo, el Partido Laborista británico, el Partido Demócrata estadounidense o el Partido Socialista español. 
en el que fuera el Partido Comunista, frente al 3\% en la Democracia Cristiana y en el Partido Socialista ${ }^{27}$. También los datos de la última década confirman una mayor atención al problema de la representación de género por parte del centro-izquierda en relación a las candidaturas y a los nombramientos, tanto en el ámbito nacional, como en el local ${ }^{28}$. En concreto, si se observa la composición del parlamento actual (que cuenta precisamente con una mayoría de centro-derecha) se hace evidente una vez más que son los partidos de izquierdas los que ofrecen una mayor presencia femenina: en la Cámara se pasa del 36,4\% de mujeres de Rifondazione Comunista, al 30\% de los Comunisti Italiani, al 28,6\% de los Verdi y el 24,3\% de los Democratici di Sinistra, frente al 10\% de la Lega Nord Padania, al 7,4\% de Forza Italia y apenas al 4\% de Alleanza Nazionale. En el Senado, donde el porcentaje de elegidas es de todas formas inferior al de la Cámara, se pasa del 20\% de mujeres de los Verdi, al 13,4\% y al 12,5\% de Margherita/Ulivo y DS/Ulivo, frente al 6,2\% de Forza Italia, al 5,9\% de la Lega Nord Padania y, de nuevo, al $2 \%$ de Alleanza Nazionale ${ }^{29}$.

Sin embargo, las investigaciones empíricas han demostrado que a menudo surge una mayor sensibilidad de los partidos hacia una representación más equitativa cuando estos partidos temen perder el consenso, y aparece en menor medida cuando el partido se siente electoralmente fuerte. He ahí que sea fundamental la capacidad de las mujeres de aliarse dentro de los diferentes partidos, incluso de forma transversal, no sólo en período electoral, sino también para ejercer una presión a más largo plazo sobre el partido, así como sobre la opinión pública y sobre los medios de información ${ }^{30}$. Un elemento de fuerza, surgido de algunas recientes experiencias político-administrativas locales, está constituido precisamente por la capacidad demostrada por algunos grupos de mujeres (generalmente pertenecientes a los partidos de izquierdas) de organizarse entre ellas y de crear una red para apoyarse durante los períodos electorales y para favorecer proyectos comunes de más larga duración, incluso en colaboración con el tejido de las organizaciones femeninas presentes en el territorio.

27. El porcentaje más alto, también en el período 1948-1992 ha sido el del Partido Radical con un $25 \%$ de elegidas que, sin embargo, en términos absolutos, dada la exigüidad numérica y de afiliación a ese partido, ha llevado al Parlamento sólo a 60 mujeres, frente a las 2887 del PCI (Pupo, Rosaria: Op. cit., p. 176).

28. En el ámbito local, la implicación de los partidos y de las coaliciones de centro-izquierda se ha manifestado sobre todo en la elección de Concejales mujeres para la composición de las Juntas (regionales, provinciales y municipales). De la situación en los Gobiernos nacionales ya hemos hablado anteriormente.

29. Datos tomados de la página web www.arcidonna.org, reelaborados a partir de los datos del Parlamento, actualizados en septiembre del 2003.

30. En el Parlamento italiano, se ha asistido recientemente a un intento -finalmente fallido- de conexión transversal entre las mujeres pertenecientes a los partidos en el gobierno y en la oposición, para crear un frente común contra la aprobación de las «Normas en materia de procreación médica asistida» convertidas en Ley n ${ }^{\circ} 40$ de 19 de febrero de 2004, que tiende a limitar la libertad de elección individual de las mujeres respecto a la maternidad responsable e introduce elementos que, en un futuro, podrían comportar una revisión de las normas que regulan la interrupción voluntaria del embarazo. 
Del análisis empírico de algunas experiencias locales nacen motivos de reflexión interesantes acerca de los factores que pueden contribuir a una mayor participación política femenina, también en los lugares de toma de decisión.

De momento, entre los casos italianos más emblemáticos en el ámbito local se encuentra la administración provincial de Prato ${ }^{31}$, que arroja en Toscana la presencia más alta de mujeres en una Junta provincial ${ }^{32}$.

Del análisis del caso de Prato, y comparando el Municipio y la Provincia, se hacen evidentes tres elementos de relieve: a) el alto grado de participación social y económica de las mujeres y, sobre todo, su visibilidad social; b) una unión bastante fuerte, dictada por la pertenencia a partidos y por la tradición de una subcultura política local, que ha mantenido unidas a las elegidas, en los Consejos, y a las nombradas, en las Juntas, de forma distinta en las dos realidades de Prato; c) la dimensión institucional que ha dado lugar a la formación de la Provincia de Prato como provincia autónoma con respecto a Florencia solamente en 1995, un hecho que ha dejado un margen de acción más amplio para las neo-elegidas y nombradas.

En relación al primer punto, la realidad local de Prato presenta una alta participación de las mujeres en la vida económica, hasta el punto de que, en 2001, el empleo femenino experimentaba un crecimiento y se consolidaba en torno al $43 \%$, un valor superior en relación a la media regional del $37 \%$, pero también en la vida social y, en concreto, en el asociacionismo (entre las participantes activas del asociacionismo femenino se encuentran a menudo aquellas que ya lo fueron en los movimientos feministas de los años setenta). Pero, sobre todo, parece determinante el grado de visibilidad y reconocimiento, por parte de la colectividad, de este protagonismo femenino. Una visibilidad que, probablemente, se origina en el recorrido trazado por algunas mujeres "pioneras» de la política de Prato, quienes ya en la posguerra desempeñaron roles de primera fila en la representación y en el gobierno de la ciudad ${ }^{33}$.

La segunda dimensión, finalmente, se entrelaza con la tercera, la más específicamente institucional: el hecho de compartir un determinado tipo de subcultura política ha ejercido como elemento aglutinante entre las primeras mujeres que entraron en el Consejo y en la Junta provincial y las ha llevado

31. Prato es un distrito industrial textil muy estudiado por los economistas del ámbito internacional. Su caracterización en este sentido no ha dejado de ser determinante en la inclusión precoz de las mujeres en el mercado del trabajo y en la sociedad, con consecuencias significativas en la participación política (BECATTINI, Giacomo: Il distretto industriale. Un nuovo modo di interpretare il cambiamento economico, Turín, Rosenberg \& Sellier, 2000).

32. Una presencia paritaria (si se excluye el Presidente de la Junta), con un salto del 16,7\% de 1995 al $50 \%$, precisamente, de 1999 y una presencia del 29,2\% de consejeras (en ligera disminución respecto al $33,3 \%$ de la legislatura anterior).

33. Pensemos, por ejemplo, en el rol desempeñado en la ciudad por Anna Fondi, dirigente de la Comisión femenina del PCI de Prato y una de las fundadoras de la Unión Mujeres Italianas, elegida consejera municipal por primera vez en 1951, cargo que le será confirmado ininterrumpidamente hasta 1973, desempeñando también el cargo de Concejal de Servicios Sociales durante doce años y creando un sistema de welfare local que abandona la orientación de tipo asistencialista para abrazar una política estructural de servicios para la persona y la familia. 
a unirse y a aliarse de cara a los objetivos reconocidos como comunes (entre otros, actuar para conseguir una mayor presencia femenina en los órganos locales $^{34}$ ); al mismo tiempo, ha desempeñado también un papel de relieve la otra dimensión, es decir, el hecho de que estas primeras mujeres hayan sido vistas actuando en una institución nueva, formada sólo en tiempos recientes. Todo ello les ha abierto a las mujeres un espacio de acción política e institucional que habría sido impensable en un contexto en el que las lógicas y las alianzas de poder estuvieran ya consolidadas $y$, por consiguiente, y por las razones ya mencionadas, estructuradas de forma masculina ${ }^{35}$.

\section{LOS POSIBLES DESARROLLOS DE LA PARTICIPACIÓN DE LAS MUJERES EN LA POLÍTICA. EN ITALIA ¿VUELTA A EMPEZAR DESDE PEKÍN? CONSIDERACIONES FINALES}

El cuadro descrito hasta ahora no es desde luego alentador, si nos limitamos a observar el número de mujeres presentes en la escena política italiana. No obstante, al retraso fundamental de las instituciones políticas nacionales no parece corresponder a una parálisis de las instituciones políticas locales que, cuanto más cercanas a la sociedad civil, reflejan las dinámicas de cambio que pueden ser puestas en marcha desde abajo, a partir de las prácticas femeninas de «hacer» política.

Muy a menudo en los últimos años se ha dado una ruptura entre la política nacional y las cúpulas de los partidos, por un lado, y los elementos participativos e innovadores emergentes en el ámbito político local y en la sociedad civil, por el otro. La dimensión de género no se ha librado de este gap y, por el contrario, ha demostrado lo amplia que puede llegar a ser la distancia entre el dirigismo de la política desde arriba y la política participativa desde abajo.

Si algunas realidades locales parecen estar adoptando estrategias innovadoras, también buena parte de la sociedad italiana se presenta ya libre, en general, de prejuicios negativos en relación con la presencia de las mujeres en la política y se muestra sensible a los problemas de una representación más equilibrada en las instituciones. Se puede afirmar, pues, que una parte de la sociedad civil se muestra más progresista y más receptiva ante los cambios de la sociedad de lo que parecen hacer ciertos órganos de las direcciones de partido ${ }^{36}$.

34. Objetivo alcanzado con el paso del $16,7 \%$ al $50 \%$ de mujeres Concejales.

35. Uno de los primeros resultados obtenidos a raíz de la ya mencionada coalición de mujeres ha consistido en la elaboración y redacción de un Estatuto de la Provincia que tiene en cuenta la dimensión de género del lenguaje, algo no irrelevante si se considera lo determinante que es el lenguaje a la hora de estructurar la forma en la que se observa y se interpreta la realidad, y si se piensa en lo poco que la comunicación política y la información en general en Italia contribuyen a crear una simbología política femenina.

36. Esto es lo que se deduce a partir de varias investigaciones entre las cuales se encuentra el último informe Censis de 2003, que examina la percepción por parte de la población italiana de la participación de las mujeres en la política local; en este caso, hasta el $76 \%$ de los entrevistados afirma que considera «la presencia de las mujeres fundamental para el buen gobierno de las administraciones públicas» (www.censis.it). También un sondeo encargado por Arcidonna al 
No obstante, si el fenómeno de la participación política no puede ser reducido a una cuestión de presencia numérica, esta última no deja de contar si se pretende dar un giro positivo al reequilibrio de la representación: como atestiguan los casos europeos más avanzados, una reflexión sobre la importancia de crear coaliciones en torno a objetivos comunes, que pongan en marcha redes entre las mujeres en la política, conlleva también la cuestión de la dimensión numérica de la minoría en los espacios de toma de decisión. De hecho, para que una acción política sea eficaz en términos de connotación de género, es necesario que la presencia de las mujeres en los organismos legislativos y en los de gobierno supere un determinado "umbral crítico", por debajo del cual dicha presencia resulta poco perceptible porque el grupo minoritario tiende a uniformizarse con los modelos dominantes ${ }^{37}$. Por debajo de un «umbral» determinado, se hace difícil incluso crear alianzas entre mujeres de diferentes partidos, puesto que lo que acaba prevaleciendo es la pertenencia a los partidos por encima de las issues relevantes vinculadas al género.

De ahí la necesidad de unos instrumentos de intervención y de acciones positivas: desde este punto de vista, el Parlamento italiano, en 2003, dio un paso hacia delante al aprobar la modificación del artículo 51 de la Constitución, abriendo, así, el camino a la promoción de acciones dirigidas a garantizar la igualdad de mujeres y hombres en el acceso a los cargos electivos y a las instituciones públicas. Es éste un paso importante ya que, como se ha tenido ocasión de observar, allá donde existen los instrumentos jurídicos de apoyo y promoción de la igualdad de oportunidades en el acceso a los espacios de poder, la sociedad civil y la política demuestran estar preparadas para tomar y aprovechar las ocasiones.

Desgraciadamente, el gap entre la política de las alturas y los elementos participativos de la base no parece ser, por ahora, susceptible de ser superado: al acercarse las elecciones europeas y las administrativas locales, asistimos, justo en este momento, a la reproducción de mecanismos y automatismos en la búsqueda de candidaturas femeninas en un sentido puramente instrumental. Por ejemplo, la ley 157 de 1999 sobre la financiación pública de los partidos, en el art. 3, prevé reembolsos electorales para aquellos partidos que se comprometan a presentar como candidatas a un mayor número de mujeres en sus listas; sin embargo, como señala la prensa, algunos partidos en el gobierno han ofrecido sus candidaturas a mujeres del mundo del espectáculo, al margen de su activismo o de su capacidad de representación, para "usarlas» como señuelo, a pesar de las protestas de las militantes implicadas dentro de las mismas orga-

sociólogo Renato Mannheimer en 2000, entre otras cosas, evidenciaba la opinión positiva de los entrevistados acerca de una presencia «más o menos igual de hombres y mujeres en el Parlamento" (el 38,2\% de los entrevistados); el 44,9\% de las mujeres declaraba que sería «muy o bastante positivo para la política del país» (www.arcidonna.org).

37. Dahlerup, Drude: "From a Small to a Larger Minority: Women in Scandinavian Politics», Scandinavian Political Studies, 4-11 (1988), pp. 275-298; también son interesantes los estudios más recientes sobre la realidad político-administrativa estadounidense, en CARroll, Susan (ed): The Impact of Women in Public Office, Bloomington, Indiana University Press, 2001. 
nizaciones de partido y con una trayectoria de años de trabajo político jamás reconocido a través de las candidaturas ${ }^{38}$.

Evidentemente -y aquí volvemos a la dimensión cultural y simbólica-, Italia está viviendo también un "clima político» especial: la conversión de la política en espectáculo y su concreción en clave de mass-media no ayudan a las mujeres, desde siempre implicadas en el terreno de lo concreto y pragmático y que experimentan dificultades de afirmación individual y de grupo en el área económica y social. La «imagen» femenina vinculada a los medios de comunicación de masas no coincide con la realidad de las mujeres italianas. En concreto, los canales de televisión -múltiples, pero no plurales, ya que están sometidos a un régimen sustancialmente de monopolio ${ }^{30}-$, como es sabido, se han uniformado en torno a un estándar comercial que busca exclusivamente beneficios, han perdido toda función propiamente pública, y transmiten una imagen social desacreditada y subordinada de las mujeres.

Para las mujeres italianas, tal vez, la búsqueda de un camino que lleve hacia una participación en la política más amplia y real debería reconsiderar seriamente la Plataforma de Pequín (1995), en la que se subrayaba la importancia estratégica del acceso a los medios de comunicación de masa para el empowerment femenino: hoy en día, en Italia parece ser éste el terreno político por excelencia en el que se contrastan intereses y valores conectados con las relaciones de poder e influyentes en el plano simbólico y cultural, además de económico.

\section{BIBLIOGRAFÍA}

ANASTASI, Antonino: "Questioni di genere: donne e rappresentanza parlamentare nell'Italia repubblicana», Foedus, 6-III (2003), pp. 35-44.

- «Questioni di genere: donna vota donna?», Foedus, 6- III (2003), pp. 60-76.

Balocchi, Michela: "Genere», en G. Bettin Lattes (ed.): Per leggere la società, Florencia, Firenze University Press, 2003, pp. 179-204.

Barazzetti, Donatella y LeCCARDI, Carmen (eds.): Genere e mutamento sociale: le donne tra soggettività, politica e istituzioni, Roma, Rubbettino, 2001.

Beccalli, Bianca (ed.): Donne in quota, Milán, Elementi/Feltrinelli, 1999.

- «La democrazia, le «quote» e la parità di diritti», La Repubblica, 9 de marzo de 2004.

Bianchi, Stella (ed.): Emily. Come eleggere più donne, Roma, Reset, 1999.

Bianco, Maria Luisa: Donne al lavoro, Turín, Scriptorium, 1997.

BALBO, Laura: Riflessioni in-attuali di una ex ministro. Pensare la politica anche sociologicamente, Catanzaro, Rubbettino, 2002.

Becattini, Giacomo: Il distretto industriale. Un nuovo modo di interpretare il cambiamento economico, Turín, Rosenberg \& Sellier, 2000.

Bimbi, Franca (ed.): Differenze e diseguaglianze. Prospettive per gli studi di genere in Italia, Bolonia, Il Mulino, 2003.

Boccia, Maria Luisa: La differenza politica. Donne e cittadinanza, Milán, Il Saggiatore, 2002 .

38. FUSANI, Claudia: "AN pronta a schierare le donne dello spettacolo», La Repubblica, 28 de marzo de 2004.

39. Sobre este punto vid. los escritos de Giovanni Sartori, entre otros Mala Tempora, Milán, Laterza, 2004. 
Bonacchi, Gabriella y Groppi, Angela (eds.): Il dilemma della cittadinanza, Bari, Laterza, 1993.

Buttafuoco, Annarita: Questioni di cittadinanza. Donne e diritti sociali nell'Italia liberale, Siena, Protagon Editori Toscani, 1997.

Carroll, Susan (ed.): The Impact of Women in Public Office, Bloomington, Indiana University Press, 2001.

Cotta, Maurizio: Classe politica e parlamento in Italia, Bolonia, Il Mulino, 1979.

David, Patrizia y Vicarelli, G. (eds.): Donne nelle professioni degli uomini, Milán, F. Angeli, 1994.

Del Re, Alisa (ed.): Donne in politica. Un'indagine sulle candidature femminili nel Veneto, Milán, F. Angeli, 1999.

- «I paradossi di genere nella rappresentanza», en Franca Bimbi (ed.): Differenze e diseguaglianze. Prospettive per gli studi di genere in Italia, Bolonia, Il Mulino 2003, pp. 215-240.

DaHlerup, Drude: «From a Small to a Larger Minority: Women in Scandinavian Politics», Scandinavian Political Studies, 4-11 (1988), pp. 275-298.

Fusani, Claudia: "AN pronta a schierare le donne dello spettacolo», La Repubblica, 28 de marzo de 2004.

Gallino, Luciano: Globalizzazione e disuguaglianze, Roma, Laterza, 2000.

LEONARDI, Laura: «Uno studio comparativo sulle élites politiche locali prima e dopo l'introduzione della L.81. Il caso fiorentino", en A. Magnier (ed): Élite e comunità. I poteri locali nella transizione italiana, Catanzaro, Rubbettino, 2001, pp. 65-88.

OKIn Moller, Susan: Le donne e la giustizia. La famiglia come problema politico, Bari, Dedalo, 1999.

Pupo, Rosaria: «Le donne nel Parlamento italiano dal 1948 al 1996. Un'indagine quantitativa» en Donatella Barazzetti e Carmen Leccardi (eds.): Genere e mutamento sociale: le donne tra soggettività, politica e istituzioni, Roma, Rubbettino, 2001, pp.173-180.

Pescarolo, Alessandra: "Troppo poche in politica: ragioni e vincoli della presenza femminile», Lettera Irpet, 12 (1999), p. 4.

PHILlips, Anne: «Democrazia e rappresentanza. Ovvero, perché il sesso dei nostri rappresentanti dovrebbe avere importanza?», en Bianca Beccalli (ed.): Donne in quota, Milán, Elementi/Feltrinelli, 1999, pp.145-168.

Rossi Doria, Anna: Diventare cittadine. Il voto delle donne in Italia, Florencia, Giunti, 1996.

SANTI, Elena: "Contratti di genere. Un'applicazione e confronti europei», en Franca Bimbi (ed.): Differenze e diseguaglianze. Prospettive per gli studi di genere in Italia, Bolonia, Il Mulino, 2003, pp. 161-188.

SARTORI, Giovanni: Mala tempora, Milán, Laterza, 2004.

Unde: Human Development Report, New York, Oxford University Press, 2003. 